\title{
Globalization of Chagas Disease: A Growing Concern in Nonendemic Countries
}

\author{
Nicole Klein, ${ }^{1,2}$ Ivy Hurwitz, ${ }^{1,2}$ and Ravi Durvasula ${ }^{1,2}$ \\ ${ }^{1}$ Department of Internal Medicine, Center for Global Health, University of New Mexico Health Science Center, \\ MSC10 5550, Albuquerque, NM 87131, USA \\ ${ }^{2}$ New Mexico VA Health Care System, 1501 San Pedro Blvd SE, Albuquerque, NM 87108, USA \\ Correspondence should be addressed to Ravi Durvasula, ravi.durvasula@va.gov
}

Received 18 July 2012; Accepted 30 August 2012

Academic Editor: Alfonso J. Rodriguez-Morales

Copyright () 2012 Nicole Klein et al. This is an open access article distributed under the Creative Commons Attribution License, which permits unrestricted use, distribution, and reproduction in any medium, provided the original work is properly cited.

\begin{abstract}
Chagas disease, caused by the parasite Trypanosoma cruzi, is a major cause of morbidity and mortality in Central and South America. Initial infection and ensuing chronic infection often go undetected in the human host. High seroprevalence of T. cruzi infection is well documented in endemic areas. Designated as "a neglected tropical disease" by the World Health Organization, rural economically disadvantaged and marginalized populations in endemic countries traditionally have the highest rates of infection. As economic hardship, political instability, and the search for opportunity spur migration of infected humans from endemic to non-endemic areas of the world, blood bank data have documented rising seroprevalence of $T$. cruzi in traditionally nonendemic areas. In these areas, T. cruzi is transmitted through blood transfusion, organ transplantation, and maternal-fetal mechanisms. Increasing awareness of large numbers of infected immigrants in nonendemic countries, and the medical care they require, has focused attention on the need for strategic programs for screening affected populations, education of healthcare providers, and provision of necessary medical services for those infected. Physicians in nonendemic countries should be able to recognize signs and symptoms of acute and chronic Chagas disease as migration and globalization increase the burden of disease in non-endemic areas.
\end{abstract}

\section{Introduction}

In 1909 a Brazilian physician, Dr. Carlos Chagas, described a parasite found in the hindgut of "vinchucas" (Triatoma infestans) as being responsible for an outbreak of acute febrile illness in workers of the Central Railroad of Brazil. He named this parasite Trypanosoma cruzi and went on to describe the lifecycle of the parasite, modes of transmission to human hosts, and both acute and chronic phases of human illness. The disease became known as American trypanosomiasis or Chagas disease.

Triatomine bugs (Triatoma, Panstrongylus, Rhodnius, and other genera), the vectors of the parasite Trypanosoma cruzi, thrive in rural areas from the southern United States to the southern tip of Argentina. In endemic areas, these insects infest traditional mud-walled human dwellings. At night they bite humans sleeping inside, defecating into the fresh bite wounds and releasing feces infected with the parasites into the human host. The incubation period of disease acquired by autochthonous transmission in the immunocompetent human host ranges from 5 to 15 days. However, incubation periods as long as 21 days have been documented with oral transmission of disease (ingestion of parasite laden feces of triatomine bugs) [1] and up to 40 days in immunocompromised hosts infected through blood transfusion or organ transplantation [2, 3]. Nonspecific symptoms of acute infection appear in 10-30\% of infected hosts [4] and include fever, malaise, anorexia, lymphadenopathy, rash, and swelling at the site of inoculation (known as a Chagoma). Because of the mild nature of the symptoms of acute Chagas disease, new infections often go unrecognized. Though mortality from the acute phase of the illness is low, 70-90\% of those infected become asymptomatic carriers of the parasite [4] and can infect others through vertical transmission from mother to child or donation of blood or organs to uninfected individuals. Over 
decades, 30\% with asymptomatic chronic parasitemia will progress to chronic disease, primarily manifested as dilated cardiomyopathy, megaesophagus, or megacolon $[5,6]$.

Initially a disease of poor rural populations in Latin America, by the 1940s, urbanization ushered Chagas disease into cities. Though autochthonous transmission (from triatomine bug to human host) is less likely in the urban environment, by the 1960s chronically infected individuals transmitted an estimated 10,000 new cases of infection per year through blood transfusion in Sao Paulo, Brazil [7]. Shortly thereafter, blood donor screening programs were instituted in Brazil and other endemic countries.

Over the 100 years since its discovery, Chagas disease has become a well-recognized cause of morbidity and mortality in Central and South America. In the 1980s there were an estimated 30 million cases of Chagas disease in endemic areas of Latin America, with an annual incidence rate of 700,000 new infections and more than 45,000 deaths per year [8]. In 2002, in Latin America, the WHO estimated the burden of Chagas disease to be as high as 2.7 times the combined burden of malaria, schistosomiasis, leishmaniasis, and leprosy [9].

In the 1990s several intergovernmental initiatives between endemic countries in Latin America were developed, focusing efforts on controlling domestic triatomine vectors of the disease in order to decrease transmission to human hosts. Through multifaceted campaigns employing widespread insecticide use, improved housing conditions, and promotion of public education, annual deaths attributed to Chagas disease fell from 45,000 in 1990 to 11,000 in 2008 $[9,10]$ and estimates of infections worldwide have fallen from 30 million in 1990 to 8 million in 2006 [9].

However, the persistence of large sylvatic reservoirs of the parasite contributes to failure to eradicate the disease entirely [11]. Ultimately, failure to eradicate sylvatic mammalian reservoirs and vectors results in new infections of humans in endemic areas. Coupled with the large numbers of chronically infected patients who are unaware of their asymptomatic carrier status, T. cruzi continues to thrive in human hosts.

Over the last 30 years, a dramatic increase in human migration from endemic areas of Latin America to Europe, North America, and the Western Pacific has significantly increased seroprevalence of T. cruzi infection outside of Latin America. Decreased barriers to global trade, international travel, and migration augment movement of vectors and infected human hosts throughout the world. Nonendemic countries which receive large populations of Latin American immigrants from Chagas disease-affected areas, through sporadic case reports and a growing body of blood bank screening literature, have begun to recognize the increasing risk of acute and chronic T. cruzi infection outside of endemic areas.

\section{Globalization and Infectious Disease}

The term "globalization" refers to the increasingly intertwined relationship between cultures, economies, and people around the world. As global relationships are forged and barriers to international commerce and exchange of goods are reduced; goods and people flow across boundaries of countries and continents. Historically, there existed many examples of the spread of infectious diseases along newly forged trade routes and in areas of exploration or colonization where two previously isolated groups of people merge into one territory.

Smallpox travelled with Columbus to Hispaniola in the 1490s, with European explorers to the Aztec empire in the 16th century and to the Native American population in the United States in the 17th century [12], devastating native communities. In the 16th century, Spanish colonization of Cuba caused a measles epidemic that killed two-thirds of the native population [13]. In these cases, native populations previously unexposed to these illnesses and thus without any level of preexisting immunity experienced deadly epidemic waves of infection.

In other instances, the movement of disease vectors with sea, land, or air shipments of cargo or as inadvertent companions to travelers, spread vectors of zoonotic diseases to new frontiers. For example, the "Black Death" (bubonic plague) spread from Asia to Europe along trade routes in the 14th century [12] both by transmission from human to human and by introduction of black rats and their associated fleas that vectored the pathogen Yersinia pestis. Rat fleas and lice infected with the agent of typhus were introduced by Balkan troops into Spain in the 15th century [14] and then quickly spread across Europe.

Chagas disease differs from smallpox, measles, bubonic plague, and typhus in many respects. It is not passed from human to human by close personal contact. Nonvectorial transmission in nonendemic areas requires receipt of infected blood or organs or congenital transmission from mother to child. As individuals with chronic Chagas disease are often asymptomatic, T. cruzi infection poses a silent risk; one that requires active screening for prevention. Similar to the spread of plague and typhus vectors through exploration and trade, triatomine bug vectors of T. cruzi have been documented in many nonendemic areas including coastal Africa, the Middle East, Southeast Asia, and the Western Pacific as early as the 16th century, likely secondary to maritime trade with Latin America. However, these insects have never been documented to carry T. cruzi, and no cases of autochthonous transmission have been reported in these nonendemic regions.

In contrast, both sylvatic mammalian reservoirs [15] and numerous species of triatomine bugs $[16,17]$ are known to carry T. cruzi in the southern United States. However, only 7 cases of suspected autochthonous transmission have been documented [18-21]. Generally, housing conditions, even in rural areas in the southern United States, do not offer the same opportunity for cohabitation of humans and reduviid bugs needed for autochthonous transmission. In addition, behavioral studies of triatomine bugs in the United States have revealed a delayed defecation after blood meals [22]. For these reasons, transmission of T. cruzi in the United States and other nonendemic countries occurs largely through nonvectorial routes, from chronically infected individuals from 
endemic areas, through donation of infected blood or organs, and through vertical transmission from mother to child.

\section{Human Migration from Endemic to Nonendemic Areas of Chagas Disease}

Human migration in our time is marked by the access to international travel. In 2001 an estimated 800 million people crossed international borders, representing a fourfold increase from the 1960s [23]. Along with increased access to international travel, expansion of global trade and explosion of information technology have increased the interconnectedness of people and cultures around the world. In this environment, the driving forces of political unrest, economic hardship, and the search for opportunity spurred an unprecedented wave of Latin American emigration from Chagas disease-endemic areas to nonendemic areas of the United States, Canada, Japan, Australia, and many European countries (primarily Belgium, Spain, France, Italy, the United Kingdom, and Switzerland) over the last 30 years as seen in Figure 1 [7]. With this wave of Latin American migration came the movement of chronically infected individuals to nonendemic areas, facilitating the transmission of $T$. cruzi by nonvectorial routes.

\section{Seroprevalence of Chagas Disease in Nonendemic Countries}

Estimation of seroprevalence of Chagas disease in nonendemic countries is a formidable task plagued by many variables. Poor access of immigrants to healthcare systems, lack of universal screening programs in many nonendemic countries with large Latin American immigrant populations, and lack of awareness amongst healthcare providers of the emerging risk of Chagas disease all contribute to a likely underestimation of the problem.

In the United States and other Chagas disease nonendemic countries, the largest screening studies of seroprevalence rely on blood bank data. These data are difficult to interpret because donor populations may not be representative of larger immigrant populations as a whole [24]. In addition, a variety of available screening tests have differing sensitivity and specificity that vary by geographic strain of T. cruzi, further contributing to the difficulty interpreting screening data. Because of these caveats, most current estimates of seroprevalence of Chagas disease in nonendemic countries are based on seroprevalence data in endemic countries in combination with immigration data in nonendemic countries. This method does not capture the significant number of undocumented immigrants in nonendemic countries and is likely considerably underestimating the problem as well. In addition, there may be significant variability in seropositivity rates based on age of immigrants. Those born before widespread eradication campaigns in Latin America in the 1970s presumably have higher seropositivity rates [25].

The following is a summary of studies estimating chronic T. cruzi infections in nonendemic countries with large Latin
American immigrant populations, including the United States, Canada, Japan, Australia, and several European countries over the last 20 years.

4.1. United States. Between 1990 and 2000 the number of legal Latin American immigrants rose from 7 million to 41 million in the United States [26]. Rising awareness of the presence of chronic T. cruzi infection in these immigrant communities in the United States led to blood bank seroprevalence studies.

Between 1994 and1998, Leiby et al. screened more than 1 million blood donors in Los Angeles and more than 180,000 in Miami with a risk assessment questionnaire that aimed to identify donors born in Chagas disease-endemic countries or with history of travel to an endemic country [27]. Of these, $7.3 \%$ of Los Angeles donors were self-identified as high risk, as did $14.3 \%$ of Miami donors. Enzyme linked immunoassay (EIA) screening with radioimmunoprecipitation assay (RIPA) confirmation showed seropositivity for T. cruzi to be 1 in 7,500 in Los Angeles, and 1 in 9,000 donors in Miami. Of note, seropositivity increased significantly yearly in Los Angeles over the study period (from 1 in 9850 in 1996 to 1 in 5400 in 1998). This increase mirrored a blood donor recruitment campaign that aimed at the Hispanic community in Southern California, launched in 1993 and which increased Hispanic first-time donors in LA from 5703 in 1991 to 13,027 in 1996 [28]. All recipients of seropositive units (18 total recipients) were evaluated. None were seropositive for T. cruzi. The authors attribute this to low rates of platelet transfusion (2 of the 18 received platelets), since these blood products are suspected to be the main vehicle of transfusionassociated Chagas disease. These data suggest that seropositivity rates in areas with large Latin American immigrant populations far exceed the national estimate of 1 in 25,000 [29].

In the United States in 2007, the Red Cross began screening all blood donors (not all units) for T. cruzi using an ELISA assay which employs the epimastigote lysate antigen. Positive tests are confirmed by RIPA. Screening of approximately $65 \%$ of the US blood supply from 43 states since 2007 has revealed 1459 RIPA confirmed positive donors as of September $2011[24,30]$. The mean seropositivity rate was $1: 25,000$ to $1: 30,000$ donors tested [31]. The highest seropositivity rates occurred in areas with larger immigrant populations from endemic countries, such as California, Florida, and Texas. In these areas, rates were as high as 1 in 5200 donors [27]. Despite this seropositivity rate, very few cases of transfusion related infection were documented. These results are difficult to interpret, as immigrant donor populations may not represent immigrant populations from endemic areas on the whole. In addition, as there had already been extensive donor screening studies performed in California prior to the institution of FDA recommended screening in 2007, which resulted in permanent deferral of positive donors from the donor pool, it is likely that the seropositivity numbers in California underestimate the true rates [24]. 


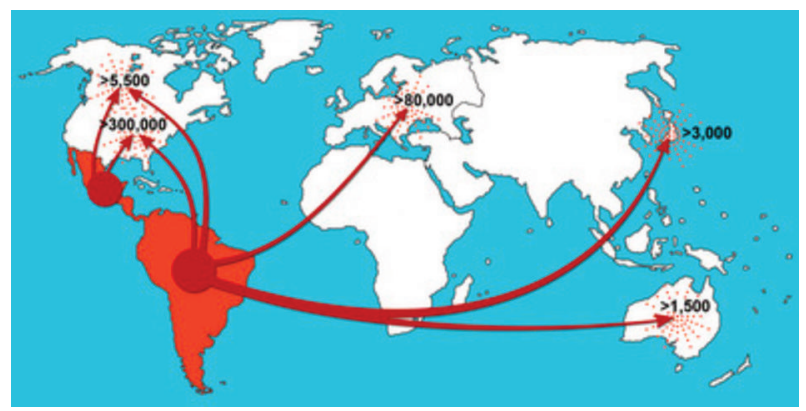

FIgURE 1: Migration routes from Latin America and estimates of Chagas disease in nonEndemic countries [7].

In 2009, based on seroprevalence in endemic Latin American countries and immigration data, Bern et al. estimated up to 300,000 immigrants in the USA to be chronically infected with T. cruzi, with an estimated 30,00045,000 cases of Chagasic cardiomyopathy, and as many of 300 congenital infections yearly [32].

4.2. Canada. In 2006, Canadian census data reveal greater than 380,000 immigrants from Mexico, Central and South America. Of the 156,960 for whom country of origin was available, 3.5\% (5553) were estimated to be infected with $T$. cruzi and 1111 likely to be in need of medical treatment for chronic disease [25]. In 2007 Steele et al. screened a small series of 102 immigrants in Canada from endemic countries in Latin America and Mexico with ELISA and reported 1\% seroprevalence rate for T. cruzi [33]. In 2010, Schmunis estimated 5,000 immigrants in Canada from T. cruzi endemic countries were chronically infected [25].

4.3. Japan. Schmunis and Yadon estimated seroprevalence rates for $T$. cruzi infection in Japan based on immigration data and endemic country rates of infection. Of more than 95,000 Latin American immigrants from Chagas endemic countries living in Japan in 2008, more than 1500 were estimated to be infected with T. cruzi [25]. However, the author notes that the actual percentage of the chronically infected may be lower, due to the generally higher socioeconomic status of these immigrants, compared to those in other nonendemic countries, and thus their lower risk of exposure prior to immigration.

4.4. Australia. In 2006 census data in Australia documented 80,522 Latin American immigrants from endemic countries residing in Australia. Of these, 3.8\% (3088) were estimated to be infected and 618 in need of medical attention [25].

4.5. Europe. Sporadic cases of Chagas disease have been recognized in Europe as early as the 1980s [34]. The International Organization of Migration reports a marked increase in migration from Latin America to Europe (primarily Southern Europe) since 2000. A concomitant increase in diagnosed cases of Chagas disease has ensued [29]. In addition to the economic recession in Latin America, there is speculation that tightening of visa requirements in the United States after 2001 and close cultural and historic ties between Latin American countries and Europe allowing some citizens to claim dual nationality due to their ancestral heritage, have contributed to the sharp rise in Latin American immigration to Europe [35].

In 2005, census data estimated 483,074 legal Latin American immigrants from Chagas endemic countries to be living in European countries (excluding Spain). Of these, $2.9 \%(14,010)$ were estimated as infected and 2803 in need of medical care [25]. By 2008 documented immigrants residing in Europe topped 38 million, with 11\% (>4 million people) hailing from Latin America [36]. In 2009, based on WHO infection rates for endemic countries and immigration data from various official sources, Basile et al. estimated that between 68,000 and 122,000 cases of Chagas disease exist in Europe, with an estimated 20 to 183 babies born annually with congenital disease (90\% of these in Spain) and a concerning projected index of undiagnosed cases being 94$96 \%[37]$.

4.6. Spain. Spain receives the highest number of Latin American immigrants of European countries. In 2003, 241,866 legal immigrants from Chagas endemic countries were reported [38]. By 2008, documented immigration to Spain from endemic Latin American countries jumped to $>1.6$ million with an estimated 5.2\% $(86,948)$ infected with T. cruzi, and $>17,000$ in need of eventual medical attention for chronic Chagas disease [25].

In 2008, Piron et al. studied 1770 at risk blood donors from endemic countries residing in Barcelona and found a $0.62 \%$ T. cruzi seroprevalence. A subset of Bolivian immigrants in this population had seropositivity rates as high as $10 \%$ [39].

In Valencia in 2005, 705 Latin American immigrants screened for T. cruzi infection showed a $1.56 \%$ seropositivity rate by hemagglutination and $0.85 \%$ positive rate by indirect immunoflourescence [40]. Another study in Valencia in 2009 and 2010 screened 1975 pregnant immigrants from 16 endemic Latin American countries, and their newborns, for antibodies to T. cruzi. Overall seroprevalence of the pregnant women was $11.3 \%$, with a subset of women from the Gran Chaco region with rates as high as $34.1 \%$. The rate of vertical transmission was 3.7\% [41]. In Madrid, a study of pregnant 
Latin American immigrants showed seroprevalence of 3.96\% [42].

In 2010, using seroprevalence rates in endemic countries and estimated numbers of Latin American immigrants, Schmunis and Yadon estimated, of Spain's more than 1.7 million immigrants, up to 87,000 may be chronically infected with T. cruzi [25].

4.7. Italy. A serology study found $1 \%$ of 99 pregnant Latin American women in Italy were seropositive for $T$. cruzi infection. $23 \%$ of 99 Bolivians tested were seropositive as were $4 \%$ of 99 adopted children and $0.6 \%$ of 155 travelers to endemic areas [43].

4.8. France. In 2007 and 2008 more than 4 million blood donations were collected through 18 different blood centers in France. 3\% (163,740 donors) were screened by questionnaire into a high-risk category for T. cruzi infection and then screened for antibodies. Seroprevalence of T. cruzi antibody was found to be 1 in 32,800 [44].

4.9. Germany. A small survey of Latin American immigrants in Berlin screened blood from 100 donors with indirect immunoflourescence (IFF), with positives subsequently screened by ELISA. Two percent seroprevalence was reported [45].

\section{Bloodbank Screening Policies in Nonendemic Countries}

Rising seropositivity rates of T. cruzi infection in nonendemic countries and the concern for transfusion transmission of infection, prompted several nonendemic countries to consider instituting blood donor screening programs over the last decade. At once weighing the concern for keeping the blood supply safe and developing policies not so stringent as to unnecessarily exclude large numbers of needed donors and jeopardize supply, various approaches and policies have been established. This issue is especially pertinent in areas where large Latin American immigrant communities make up a significant segment of the total population, including the United States. In these areas, as Latin American communities continue to grow, their contribution to the blood donor pool will become more and more necessary to sustain supply.

Generally, two different approaches to blood donor screening have been employed. The first entails a questionnaire designed to identify donors considered as a high risk (from endemic areas, with known triatomine bug exposure, or with travel history to endemic areas) and then excludes them from the donor pool. This approach has the advantage of low cost and the potential disadvantage of excluding large numbers of donors who may not be seropositive or missing donors who are seropositive without known risk factors.

The second approach to screening tests all donors with blood assays and excludes only those who are repeatedly seropositive or confirmed seropositive by a separate assay.
This approach is expensive and plagued by variable sensitivity and specificity of different screening assays and by variable specificity of the assay antigens that represent geographic strains of T. cruzi [46].

A third screening approach combines the two strategies above, identifying "high risk" donors by questionnaire and then performing screening assays on only those patients.

The European Union has no mandatory screening policy. However, several individual countries have adopted screening based on recommendation of the European Parliament in 2006 [35].

The French Blood Service (Etsablissement Francais du Sang) started screening at risk blood donors for T. cruzi antibodies in May 2007. "At risk donors", identified by questionnaire, are defined as those from endemic areas, born to a mother from an endemic area, or with history of travel to or living in endemic area. At risk donors are screened with two ELISA tests, which differ in their antigens. For double positive or discordant result, confirmation is performed with an immunoflourescence assay.

In Spain, a Royal Decree issued in 2005 permanently defers donors with positive T. cruzi serology. Immigrants from endemic areas, children of mothers from endemic areas, and those who received transfusions in endemic areas must be screened. If no serology testing is available, these donors are then excluded [25]. Some areas (Valencia and Catalonia) screen pregnant Latin American women [37].

Both the United Kingdom and Italy prohibit donation by immigrants of endemic countries [37].

In Canada blood and organ are excluded for known $T$. cruzi infection. The use of a screening questionnaire was implemented in 2009 and 2010 in the two main blood agencies [47].

In the United States, the FDA approved the Enzyme Immunoassay (EIA) for screening for T. cruzi antibody in 2006, prompting FDA Blood Products Advisory Committee (BPAC) to recommend screening blood donors. The American Red Cross and Blood Systems Inc., the two main blood donation agencies, instituted screening of all donors (not all units) in early 2007 [48] by EIA. However, serologic studies based on blood bank data in the USA since 2007 revealed low level of transfusion-related transmission, prompting the FDA to release a statement in December 2010 endorsing one time testing of all donors [31].

Regarding organ donor screening, Schwartz et al. surveyed all 58 organ procurement organizations in the United States in 2011. Of these, 11 (19\%) currently screen organ donors for T. cruzi; 4 perform a serologic screen of all donors and 7 use a risk based screening strategy. During 2008, 9 of 993 organ donors screened were positive for T. cruzi antibody. Of these 9, 6 were tested by a second method and 4 confirmed repeatedly positive [49].

\section{What Is the Risk of Transmission of T. Cruzi Parasite through Nonvectorial Routes?}

Though whole blood, packed red blood cells, platelets, leukocytes, fresh frozen plasma, and cryoprecipitate have 
all been suspected to transmit T. cruzi infection in the past [50], current evidence suggests otherwise. In 2012 Benjamin et al. examined all previously reported cases of transfusion-transmitted T. cruzi infection, the United States Canada, and Spain. Twenty case reports were examined, 11 of which were determined to represent definite transfusion-related infection [31]. In all 11 cases, apheresis or whole blood derived platelets (including leukoreduced and irradiated units) were implicated. All cases of infection occurred in immunocompromised patients. No cases of definite transfusion-related infection secondary to receipt of red blood cells, frozen plasma, or cryoprecipitate were documented.

It is interesting to note that despite these findings which implicate platelet donation in transfusion-associated T. cruzi infections, several other authors have reported that leukoreduction by centrifugation or filtration does not eliminate T. cruzi from red blood cells, buffy coat, or platelet units [51,52].

In addition, Benjamin et al. compiled data from 350 recipients of blood products from known seropositive donors in North America and Spain. Of these, 6 transfusionassociated infections were found, yielding a seroconversion rate of $1.7 \%$ (95\% CI, $0.7-3.5 \%)$ [31]. This rate is significantly lower than those previously reported in endemic areas: $12-20 \%$ seroconversion documented in Argentina, Brazil, and Chile and up to $48 \%$ in Bolivia [50].

Proposed explanations for this discrepancy include risk of transmission varying by geographic strains of $T$. cruzi and by levels of associated parasitemia [53]. Additionally, prolonged cold storage of RBC units in modern blood banks prior to transfusion may reduce transmissibility [31]. Acute infection may be difficult to diagnose, as seroconversion (especially in immunocompromised patients) may not occur early in disease, and parasitemia may not be immediately detectable during acute illness. Failure to detect subclinical transfusion-transmitted cases in immunocompetent patients with mild nonspecific symptoms may result in underreporting [50]. The latter may be especially true in nonendemic countries because seroconversion can occur as long as 90 days after transfusion [3]. Furthermore, difficulty in recognizing symptoms and making a diagnosis may worsen the situation for physicians who are unfamiliar with acute Chagas disease or the risks of transfusion-associated infection.

Because of the low documented levels of transmission of T. cruzi infection from seropositive donor units to seronegative recipients, Spain, Canada, and the USA have all restricted testing policies for blood donors. Both Spain and Canada test only donors who screen positive for risk factors by questionnaire. The USA has not adopted this policy because a study by Custer et al. found that only $75 \%$ of confirmed T. cruzi infections were identified as "at risk" donors by questionnaire or donor history [54]. The USA has thus adopted a one-time testing for all donors policy, recommended by the FDA.

From these data, the risk of transfusion-associated infection in the United States appears to be low, despite the large population of documented seropositive immigrants.

\subsection{Organ Transplant}

6.1.1. Solid Organ. Acute T. cruzi infection in solid organ transplant recipients has been well documented in Latin America [55]. In fact, due to high seroprevalence in endemic areas and shortage of donor organs, seropositivity does not preclude organ donation in many Latin American countries [56]. Transplantation of seropositive organs to seronegative donors with subsequent seroconversion and/or acute infection in heart, kidney, and liver transplant recipients in endemic countries has been documented. In 2011, Pinazo et al. published recommendations for management of Chagas disease in transplant patients [57].

Transplant-associated transmission of T. cruzi has also been documented in nonendemic countries. For example, five cases have been reported in the United States. In 2001, three organ transplant recipients (37-year-old female kidney and pancreas recipient, 32-year-old female liver recipient, and 69-year-old female kidney recipient) from the same donor (a Central American immigrant), all contracted acute Chagas disease. All three patients were treated with nifurtimox. One survived with no evidence of recurrent disease. The other two died: one of acute Chagasic myocarditis and the other of unrelated sepsis [55]. Two other cases have been reported in the United States. In 2005, a 64year-old male heart transplant recipient developed acute $T$. cruzi parasitemia one year after transplant. He was treated successfully for T. cruzi infection but later died of rejection. Three other organ recipients from the same donor were seronegative by IFA for T. cruzi, with no evidence for parasitemia by PCR [58]. In 2006, a 73-year-old male heart transplant recipient developed acute T. cruzi infection one month after transplant. He was treated with nifurtimox with resolution of parasitemia, but later died of cardiac failure [58]. In another instance, an organ donor from El Salvador was RIPA antibody positive for T. cruzi. Three other organ recipients from this donor were all negative for T. cruzi infection.

In Spain, of 1500 transplants performed in 2006, 10\% were from foreign organ donors and $3 \%$ were foreign recipients. Forty percent of nonnative donors were Latin American, from countries endemic for Chagas disease. The Spanish National Organization for Transplantation, recognizing the surge in Latin American immigration over the last decade, and the accompanying increase in seroprevalence in Chagas disease, has now instituted mandatory donor screening for donors at risk [56].

Reactivation of chronic Chagas disease in chronically infected organ recipients, likely secondary to heavy immunosuppression, is also a consideration in both endemic and nonendemic areas. Acute reactivation of disease has been observed in chronically infected heart and kidney transplant patients [56]. Chronically infected recipients of heart transplants appear to be at significant risk, with acute reactivation reported in $20-30 \%[59,60]$. Similarly, reactivation of chronic disease has been observed in other forms of immunosuppression, including HIV or chemotherapy.

Also a consideration is the burgeoning transplant tourism industry, estimated to be worth $\$ 60$ billion dollars 
worldwide in 2006 [61]. As noted above, in many endemic countries, because of high levels of seroprevalence of $T$. cruzi and the need for donor organs, seropositivity is not a contraindication to organ donation. Organ recipients transplanted in Chagas endemic countries are at risk of returning to their native countries with acute transplantationassociated Chagas disease.

6.2. Oral. In endemic countries, outbreaks of acute Chagas disease acquired through oral consumption of food contaminated with triatomine feces or ground triatomine bugs have been documented. Notably, in the last ten years, 138 outbreaks of orally transmitted infection have been recognized in the Brazilian Amazon, where triatomine domiciliary and peridomestic control has been effective [62]. Outbreaks in Brazil have been linked to contaminated sugar cane juice [63-65], acai juice and acai paste [66], water [1], or bacaba (a regional fruit) [67]. In Columbia contaminated fresh orange and tangerine juice have been implicated [68]. In Venezuela, a large outbreak in school children was linked to consumption of contaminated guava juice [69]. Host susceptibility to infection through oral exposure is dependent upon burden of ingested parasites, resistance of specific species of parasite to the host's gastric environment, and the host's local immune response [62]. To date, no outbreaks of orally transmitted Chagas disease have been reported in nonendemic countries. However, in nonendemic countries where infected vectors are known to be present, particularly the American South, risk of oral transmission is theoretically present. Perhaps the lack of similar foraging behaviors of humans in sylvatic triatomine environments and more widespread use of pasteurization have prevented oral transmission in this environment.

6.3. Congenital. Vertical transmission from a chronically infected mother to the fetus occurs at a rate of approximately $5 \%$ [70-72]. Risk of transplacental transmission may be as high as $70 \%$ in acute T. cruzi infection acquired by the mother during pregnancy [73]. Risk factors for congenital transmission include multiple pregnancies [74], high maternal parasitemia during acute phase, maternal confection with HIV, and low immune response to infection in mother or child [75].

In endemic countries with the highest burden of Chagas disease, such as Bolivia, Brazil, and Paraguay, seropositivity in pregnant women has been estimated to be as high as 25$51 \%$ [76]. An estimated 43 to $54 \%$ of congenitally infected infants will be symptomatic [72]. Newborns with acute $T$. cruzi infection may have lower APGAR scores, gestational ages, birth weights and lengths, and head circumferences than babies of mothers who are not infected [72]. Two studies in Argentina and Chile suggested increased rates of miscarriage with maternal T. cruzi infection [77-79].

Recent trends in immigration from Latin America to Europe show an increasingly young, well-educated, and female immigrant population [80]. In this community, congenital transmission likely represents the highest risk of new T. cruzi infections in nonendemic countries.
Di Pentima et al. screened 2107 Hispanic and 1658 nonHispanic pregnant women who presented to a community health clinic in Houston, Texas, for T. cruzi antibody between 1993-1996. Positive ELISA screening assay was confirmed by hemagglutination assay. A $0.4 \%$ prevalence in Hispanic women and $0.1 \%$ prevalence in non-Hispanic women were reported, illustrating the risk of congenital transmission in the United States [81].

Two studies estimated numbers of undiagnosed cases of congenital transmission yearly in the United States, based on seropositivity studies of pregnant Latin American immigrants, to range between 63 and 635 per year $[32,82]$.

Recognized cases of congenital transmission of T. cruzi outside of endemic areas include one in Spain born to a Bolivian mother, one in Sweden [34], one in Switzerland [83], and several possible cases in the USA [84, 85]. This illustrates the degree of underdiagnosis of congenital infection in nonendemic countries.

Treatment of acute Chagas disease (including infection of newborns) with either nifurtimox or benznidazole results in cure of parasitemia in approximately $50 \%$ of cases [86, 87]. For this reason, detection of seropositive mothers and monitoring for acute T. cruzi infection in their newborns offers a valuable window of opportunity to treat and cure Chagas disease. In contrast, as infection progresses to indeterminate and chronic phases, treatment of Chagas disease is controversial due to poor tolerability of medications and unclear clinical benefit [88]. Various studies have shown possible benefit with treatment of specific groups of patients in indeterminate phase or chronic infection. Notably, the ongoing BENEFIT trial is currently evaluating reduction in morbidity and mortality with treatment of chronic Chagasic cardiomyopathy with benznidazole in subgroups of patients.

Given the highest cure rate of Chagas disease with treatment during acute infection, detection of congenital transmission, either by maternal or newborn screening, offers a unique opportunity to intervene and ultimately decrease the burden of morbidity and mortality associated with development of chronic infection. Prenatal screening for other often asymptomatic chronic infectious diseases, such as hepatitis B and HIV, is required in many US states [89]. Public health authorities should consider similar screening of at risk pregnant women for $T$. cruzi infection in nonendemic areas with large Latin American immigrant communities.

\section{Burden of Chronic Cardiomyopathy Patients in Nonendemic Countries}

Chagasic myocarditis is characterized by a dilated cardiomyopathy, apical cardiac aneurysms, and intracardiac thrombi. Patients are at risk of sudden death from acute arrhythmias or pulmonary emboli. Storino and Milei studied the evolution of Chagasic cardiomyopathy in endemic and nonendemic areas over a 5-12, year period. In endemic areas, $43 \%$ of chronically infected patients developed Chagasic cardiomyopathy versus only $7 \%$ in nonendemic areas. This difference was attributed to repeated exposure to the parasite 
in endemic areas [90]. For this reason, it is difficult to estimate the number of patients projected to develop Chagasic cardiomyopathy in nonendemic countries, and what their healthcare needs will be.

Based on Schmunis and Yadon's estimates of seroprevalence of T. cruzi infection in Europe [25], Guerri-Guttenberg et al. estimated between 1680 and 8284 patients with chronic Chagasic cardiomyopathy in Europe in 2008, with the largest proportion of these residing in Spain and Italy [91]. As only a handful of cases of Chagasic cardiomyopathy have been reported in Europe [91], it is likely this condition is widely underdiagnosed.

In 2010, based on immigration data and known seroprevalence in endemic countries, Schmunis estimated more than 49,000 patients with Chagasic cardiomyopathy residing in the United States [25].

As these patients age, disease manifestations become progressively more severe, and patients require medical attention. In areas of large Latin American immigrant communities, significant healthcare resources will likely be needed to diagnose and care for these patients.

\section{Diagnostic Challenge}

In nonendemic countries, in order to prevent transfusion or organ donation associated transmission of Chagas disease, to treat congenitally acquired acute infections, and to provide access to healthcare for those with chronic manifestations of disease, access to appropriate diagnostic testing is essential. Current access is not optimal, with routine serologic tests often difficult to obtain and subsequently requiring prolonged waiting periods to obtain results. In addition, healthcare providers in nonendemic areas may not be familiar with appropriate testing methods necessary to diagnose various stages of $T$. cruzi infection.

For example, during acute infection the more widely available serologic testing for T. cruzi antibodies, enzyme linked immunosorbent assay (ELISA), indirect immunoflourescent antibody (IFA), radioimmunoprecipitation assay (RIPA), indirect hemagglutination (IHA), and complement fixation are often negative because the host's antibody response has not yet reached detectable levels. During the acute stage, direct identification of parasites on blood smear may be difficult for pathologists or microbiologists in nonendemic countries who are unfamiliar with the disease. Furthermore, diagnosis by PCR is only readily available in research settings, providing further diagnostic challenge [92].

Indeterminate or chronic stages of Chagas disease, often marked by low levels of parasitemia, are more readily diagnosed by serologic antibody testing. However, serologic tests are plagued by varying levels of sensitivity which differ based on regional genetic variation in T. cruzi species, crossreactivity (mostly with leishmania, malaria, syphilis, or in collagen vascular diseases), and false negative results [93].

Not only must a nonendemic provider think to include Chagas disease in the differential diagnosis in the appropriate settings, but he or she must also have an understanding of and access to suitable diagnostic methods.

\section{Healthcare Provider Awareness of Risk of T. Cruzi Infection in Nonendemic Countries}

In 2008 Medscape CME, in conjunction with the Centers for Disease Control and Prevention (CDC), conducted a "Knowledge, Attitudes and Practices" (KAP) assessment of physicians accessing its website. The results of five 10question surveys, each aimed at a specific group of specialists (including Infectious Disease, Cardiology, Obstetrics and Gynecology, and Transplant Medicine) or general practitioners, assessed general awareness of Chagas disease, consideration of risk for Chagas disease in practice, and knowledge of clinical aspects of the disease. Results showed varying familiarity with the disease across specialties [94].

Concerning is the finding that Chagas awareness was lowest among obstetricians and gynecologists, who have a unique opportunity to recognize and intervene in cases of congenital infection. $60 \%$ of 292 obstetricians and gynecologists surveyed, reported never considering the diagnosis in their practice [94]. Similarly, of 125 transplant physicians surveyed, $25 \%$ reported never having heard of Chagas disease and 39\% reported never considering the risk of Chagas disease in their patients. Among 280 cardiologists surveyed, $34 \%$ reported never considering the risk of Chagas disease in their patients. These numbers illustrate the wide knowledge gap likely present in providers of many nonendemic countries which needs to be addressed by education and awareness programs.

\section{Treatment Challenge}

Even if a healthcare provider is able to diagnose a case of Chagas disease in a nonendemic country, obtaining appropriate treatment remains an obstacle. Benznidazole, the primary drug used to treat Chagas disease, is available primarily in endemic countries. Even in endemic countries, a recent production shortage has left thousands of patients without access to treatment [95]. A second drug, nifurtimox, is only commercially available in a handful of countries. In most nonendemic countries neither benznidazole nor Nifurtimox is widely available. For example, in United States, where neither drug is approved by the FDA, they are only available by special request from the CDC through investigational protocols for compassionate treatment. In 2010 , the CDC reported in only $11 \%$ of cases of that more than 500 individuals identified as T. cruzi seropositive by blood donation screening had a physician or patient contacted the CDC for consultation for treatment [94]. An international drug and research organization, Drugs for Neglected Diseases initiative (DNDi), conducts a Chagas disease program focused on clinical development of existing antifungal drugs for new use in combination therapy with existing treatments for Chagas disease and on new drug development. 


\section{Political Issues Surrounding Chagas Disease in Nonendemic Areas}

Challenges in diagnosing and treating Chagas disease in nonendemic countries include funding healthcare provider education programs, providing screening programs for pregnant women, blood and organ donors, and ensuring access to healthcare for chronically infected individuals. However, a multitude of socioeconomic factors in nonendemic countries that receive immigrants, and in immigrant populations themselves, contribute to the difficulties in reaching ability at risk populations and providing culturally appropriate care.

General barriers to immigrants obtaining healthcare include cultural and language barriers, lack of regular healthcare provider, lack of access to affordable insurance or healthcare, lack of information, job constraints, lack of trust in government programs, and fear. For example, in 2002 Doty and Ives found 40\% of Hispanics in the United States (versus $20 \%$ of whites) are without a regular healthcare provider [96].

In 2010, after a surge in Latin American immigration to Italy, Di Girolamo et al. studied attitudes of undocumented Latin Americans in Bologna towards healthcare by reviewing records from two medical clinics which primarily serve undocumented immigrants [97]. They concluded that the well-documented lack of access to medical diagnosis and care for underserved, poor, and rural populations at risk for Chagas disease in endemic countries [98], and the social stigmatization of this disease in endemic countries based on its association with poor and socially marginalized populations [99] contribute to lack of diagnosis of Chagas disease in many immigrants priorto immigration from endemic to nonendemic countries. For this reason, immigrants from endemic areas may be unaware of their diagnosis, or because of the stigma and the practice of exclusion from the labor market in endemic countries, may be unwilling to acknowledge their diagnosis and access medical care [100].

In addition, Di Girolamo et al. theorized that the economic hardship in Europe during this period of Latin American immigration, and the 2002 Italian immigration policy requiring employment in order to qualify for a residency permit, caused undocumented immigration in Europe to become widespread [97]. Furthermore, when undocumented immigration became illegal in Italy in 2009, a subsequent $50 \%$ decrease in volume in migrant healthcare clinics was observed [101]. Interviews with members of the Latin American immigrant population in the area revealed that due to the stigma of poverty and ignorance associated with Chagas disease in their native countries, many migrants distance themselves from health interventions targeted to this disease specifically, fearing it may promote anti-immigration policies [97].

The synthesis of these data prompted Di Girolamo et al. in agreement with a previous mandate from the WHO Commission on Social Determinants of Health, call for a multifaceted approach which integrates biopsychosocial elements of the disease into a comprehensive intervention strategy targeting at risk populations while taking into account the cultural context of the disease.
Such programs must take into account the social, cultural, and economic aspects of the disease to improve access to medical care for at risk populations, implement effective surveillance, enforce monitoring of blood and organ donors, and detect and treat congenital infections. Most importantly, healthcare providers in nonendemic countries with large immigrant populations from Chagas diseaseendemic areas need to be educated about the existence of the disease, its clinical manifestations, and the appropriate mechanisms of diagnosis and treatment of infected patients. Specifically, healthcare providers in the fields of obstetrics and gynecology, transplant medicine, hematology/oncology (immunocompromised patients receiving multiple blood transfusion products), cardiology, gastroenterology, and infectious disease, and those who provide care in immigrant communities should be the focus of education programs. In particular, a strong focus should be on detection of and treatment of congenitally acquired infections, as this intervention offers the highest medical cure rate and can in the long term lead to decrease in burden of chronic disease.

\section{What Is Being Done about Chagas Disease in Nonendemic Countries?}

In the United States, the Centers for Disease Control (CDC) Division of Neglected Parasitic Diseases partners with local and state health departments to (1) educate healthcare providers about Chagas (2) and increase provider awareness by providing free CME (3) study perceptions and awareness of Chagas disease in patients and healthcare providers in order to improve education and outreach programs, decrease barriers to accessing care, and (4) aid in global efforts to control transmission of the disease via vectors and congenital means [102].

In 2007 the WHO announced the WHO Global Network for Chagas Elimination, after a rise in detected cases in nonendemic countries. Stated goals of the Network are to increase surveillance, decrease transfusion and transplantation related infections in endemic and nonendemic countries, expand prevention of congenital transmission and recognition and treatment of newborn disease, and identify appropriate screening and diagnostic tests [103].

The Nonendemic Countries Initiative is one of the first initiatives produced by the Network. Belgium, France, Italy, Spain, Switzerland, the United Kingdom, Northern Ireland, Japan, and the United States are all participants [9]. A working group on Chagas disease in Europe recommends the following approach:

(i) creation of international information and surveillance systems to track reported cases, control transmission and to educate healthcare providers;

(ii) definition of the risk of congenital transmission in Latin American immigrants;

(iii) consideration of systemic screening of blood, organ, and tissue donors from endemic countries;

(iv) facilitating access to diagnosis and treatment for migrant groups at risk; 
(v) expanding education about tropical diseases and international health in European universities [37].

\section{Conclusion}

A sharp increase in migration from Chagas endemic countries of Central and South America to nonendemic countries of North America, the Pacific basin, and Europe has transformed Chagas disease into a global medical challenge. Current estimates of seroprevalence of $T$. cruzi infection in nonendemic countries are likely grossly underestimated due to large undocumented populations. Lack of diagnosis and screening for infected individuals allows propagation of disease by nonvectorial routes of maternal-child transmission and receipt of infected blood products or organs. Given the social, political, and economic aspects of this disease in endemic countries, comprehensive outreach programs aimed at engaging migrant populations and educating healthcare providers are necessary to prevent spread of disease and obtain timely treatment for those infected.

\section{Acknowledgment}

This work was supported by NIH/NIAID RO1AI66045 to R. Durvasula.

\section{References}

[1] J. P. Dias, C. Bastos, E. Araújo et al., "Acute Chagas disease outbreak associated with oral transmission," Revista da Sociedade Brasileira de Medicina Tropical, vol. 41, no. 3, pp. 296-300, 2008.

[2] Centers for Disease Control and Prevention (CDC), "Blood donor screening for Chagas disease-United States, 20062007," Morbidity and Mortality Weekly Report, vol. 56, no. 7, pp. 141-143, 2007.

[3] D. A. Leiby, B. A. Lenes, M. A. Tibeals, and M. T. TamesOlmedo, "Prospective evaluation of a patient with Trypanosoma cruzi infection transmitted by transfusion," New England Journal of Medicine, vol. 341, no. 16, pp. 1237-1239, 1999.

[4] L. V. Kirchhoff, "American trypanosomiasis (Chagas disease)," in Tropical Infectious Diseases: Principles, Pathogens \& Practice, R. Guerrant, D. H. Walker, and P. F. Weller, Eds., p. 1082, Churchill Livingstone, Philadelphia, Pa, USA, 2nd edition, 2006.

[5] J. H. Maguire, R. Hoff, and I. Sherlock, "Cardiac morbidity and mortality due to Chagas' disease: prospective electrocardiographic study of a Brazilian community," Circulation, vol. 75, no. 6, pp. 1140-1145, 1987.

[6] J. C. Pinto Dias, "Natural history of Chagas disease," Arquivos Brasileiros de Cardiologia, vol. 65, no. 4, pp. 359-366, 1995.

[7] J. R. Coura and P. A. Vĩas, "Chagas disease: a new worldwide challenge,” Nature, vol. 465, no. 7301, pp. S6-S7, 2010.

[8] J. C. P. Dias, A. Prata, and D. Correia, "Problems and perspectives for Chagas disease control: in search of a realistic analysis," Revista da Sociedade Brasileira de Medicina Tropical, vol. 41, no. 2, pp. 193-196, 2008.

[9] Secretariat of World Health Organization, Chagas Disease: Control and Elimination, Secretariat of World Health Organization, 2009.
[10] F. Guhl, N. Pinto, and G. Aguilera, "Sylvatic triatominae: a new challenge in vector control transmission," Memorias do Instituto Oswaldo Cruz, vol. 104, supplement 1, pp. 71-75, 2009.

[11] D. T. John and K. L. Hoppe, "Trypanosoma cruzi from wild raccoons in Oklahoma," American Journal of Veterinary Research, vol. 47, no. 5, pp. 1056-1059, 1986.

[12] The Mariners Museum, http://ageofex.marinersmuseum .org $/$ ?type $=$ webpage $\&$ id $=48$.

[13] J. P. Byrne, Encyclopedia of Pestilence, Pandemics, and Plagues, Greenwood Publishing Group, Westport, Conn, USA, 2008.

[14] L. Altman, Plague and Pestilence: A History of Infectious Disease, Enslows Publishers, Berkeley Heights, NJ, USA, 1998.

[15] E. L. Brown, D. M. Roellig, M. E. Gompper et al., "Seroprevalence of Trypanosoma cruzi among eleven potential reservoir species from six states across the southern United States," Vector Borne and Zoonotic Diseases, vol. 10, no. 8, pp. 757763, 2010.

[16] C. E. Reisenman, G. Lawrence, P. G. Guerenstein, T. Gregory, E. Dotson, and J. G. Hildebrand, "Infection of kissing bugs with Trypanosoma cruzi, Tucson, Arizona, USA," Emerging Infectious Diseases, vol. 16, no. 3, pp. 400-405, 2010.

[17] S. A. Kjos, K. F. Snowden, and J. K. Olson, "Biogeography and Trypanosoma cruzi infection prevalence of chagas disease vectors in Texas, USA," Vector-Borne and Zoonotic Diseases, vol. 9, no. 1, pp. 41-49, 2009.

[18] T. R. Navin, R. R. Roberto, and D. D. Juranek, "Human and sylvatic Trypanosoma cruzi infection in California," American Journal of Public Health, vol. 75, no. 4, pp. 366-369, 1985.

[19] D. E. Ochs, V. S. Hnilica, D. R. Moser, J. H. Smith, and L. V. Kirchhoff, "Postmortem diagnosis of autochthonous acute chagasic myocarditis by polymerase chain reaction amplification of a species-specific DNA sequence of Trypanosoma cruzi," American Journal of Tropical Medicine and Hygiene, vol. 54, no. 5, pp. 526-529, 1996.

[20] B. L. Herwaldt, M. J. Grijalva, A. L. Newsome et al., "Use of polymerase chain reaction to diagnose the fifth reported US case of autochthonous transmission of Trypanosoma cruzi, in Tennessee, 1998," Journal of Infectious Diseases, vol. 181, no. 1, pp. 395-399, 2000.

[21] P. L. Dorn, L. Perniciaro, M. J. Yabsley et al., "Autochthonous transmission of Trypanosoma cruzi, Louisiana," Emerging Infectious Diseases, vol. 13, no. 4, pp. 605-607, 2007.

[22] S. A. Klotz, P. L. Dorn, J. H. Klotz et al., "Feeding behavior of triatomines from the southwestern United States: an update on potential risk for transmission of Chagas disease," Acta Tropica, vol. 111, no. 2, pp. 114-118, 2009.

[23] M. Cetron, " The world and its moving parts," in Institute of Medicine Workshop on the Impact of Globalization on Infectious Disease Emergence and Control: Exploring the Consequences and Opportunities, Institute of Medicne Forum on Emerging Infections, Washington, DC, USA, 2002.

[24] C. Bern and S. P. Montgomery, "An estimate of the burden of chagas disease in the United States," Clinical Infectious Diseases, vol. 49, no. 5, pp. e52-e54, 2009.

[25] G. A. Schmunis and Z. E. Yadon, "Chagas disease: a Latin American health problem becoming a world health problem," Acta Tropica, vol. 115, no. 1-2, pp. 14-21, 2010.

[26] US Census Bureau, United States Foreign-Born Population, 2000.

[27] D. A. Leiby, R. M. Herron, E. J. Read, B. A. Lenes, and R. J. Stumpf, "Trypanosoma cruzi in Los Angeles and Miami blood 
donors: impact of evolving donor demographics on seroprevalence and implications for transfusion transmission," Transfusion, vol. 42, no. 5, pp. 549-555, 2002.

[28] Y. Wu, S. A. Glynn, G. B. Schreiber et al., "First-time blood donors: demographic trends," Transfusion, vol. 41, no. 3, pp. 360-364, 2001.

[29] G. A. Schmunis, "Epidemiology of Chagas disease in nonendemic countries: the role of international migration," $\mathrm{Me}$ morias do Instituto Oswaldo Cruz, vol. 102, no. 1, pp. 75-85, 2007.

[30] American Associated of Blood Banks, "AAB Chagas' disease biovigilance network," 2011, http://www.aabb.org/programs/biovigilance/Pages/chagas.aspx.

[31] R. J. Benjamin, S. L. Stramer, D. A. Leiby, R. Y. Dodd, M. Fearon, and E. Castro, "Trypanosoma cruzi infection in North America and Spain: evidence in support of transfusion transmission," Transfusion, vol. 52, no. 9, pp. 1913-1921, 2012.

[32] C. Bern, S. Kjos, M. J. Yabsley et al., "Trypanosoma cruzi and Chagas' disease in the United States," Clinical Microbiology Reviews, vol. 24, no. 4, pp. 655-681, 2011.

[33] L. S. Steele, D. W. MacPherson, J. Kim, J. S. Keystone, and B. D. Gushulak, "The sero-prevalence of antibodies to Trypanosoma cruzi in Latin American refugees and immigrants to Canada," Journal of Immigrant and Minority Health, vol. 9, no. 1, pp. 43-47, 2007.

[34] P. O. Pehrson, M. Wahlgren, and E. Bengtsson, "Asymptomatic congenital Chagas' disease in a 5-year-old child," Scandinavian Journal of Infectious Diseases, vol. 13, no. 4, pp. 307-308, 1981.

[35] P. Albajar-Vinas and J. Jannin, "The hidden Chagas disease burden in Europe," Euro Surveillance, vol. 16, no. 38, 2011.

[36] K. Vasileva, "Statistics in focus. Citizens of European countries account for the majority of the foreign population in EU-27 in 2008," Eurostat 94/2009, Luxembourg, Luxembourg, 2009.

[37] L. Basile, J. M. Jansa, Y. Carlier et al., "Chagas disease in European countries: the challenge of a surveillance system," American Journal of Tropical Medicine and Hygiene, vol. 81, no. 6, pp. 927-933, 2009.

[38] J. Gascon, "Diagnosis and treatment of imported Chagas disease," Medicina Clínica, vol. 125, no. 6, pp. 230-235, 2005.

[39] M. Piron, M. Vergés, J. Muñoz et al., "Seroprevalence of Trypanosoma cruzi infection in at-risk blood donors in Catalonia (Spain)," Transfusion, vol. 48, no. 9, pp. 1862-1868, 2008.

[40] C. Parada, E. Vila, and J. Villaba, "Determinacion de Trypanosoma cruzi: estudio preliminar de prevalencia en donantes de sangre," Tech. Rep., Centro de Transfusion, Comunidad Valenciana, Resumen del Congreso SETS, Sa Sebastian, Spain, 2005.

[41] C. Barona-Vilar, M. J. Giménez-Martí, T. Fraile et al., "Prevalence of Trypanosoma cruzi infection in pregnant Latin American women and congenital transmission rate in a non-endemic area: the experience of the Valencian Health Programme (Spain)," Epidemiology \& Infection, vol. 140, no. 10, pp. 1896-1903, 2012.

[42] M. D. Flores-Chavez, F. J. Merino, S. Garcia-Bujalance et al., "Surveillance of Chagas disease in pregnant women in Madrid, Spain, from 2008 to 2010," Euro Surveillance, vol. 16, no. $38,2011$.

[43] M. Anselmi, A. Angheben, and M. Degani, "Imported Chagas disease in italy: preliminary screening results of selected immigrant populations," Tropical Medicine \& International Health, vol. 14, supplement 2, p. 74, 2009.

[44] A. Assal and C. Corbi, "Chagas disease and blood transfusion: an emerging issue in non-endemic countries," Transfusion Clinique et Biologique, vol. 18, no. 2, pp. 286-291, 2011.

[45] M. Frank, B. Hegenscheid, K. Janitschke, and T. Weinke, "Prevalence and epidemiological significance of Trypanosoma cruzi infection among Latin American immigrants in Berlin, Germany," Infection, vol. 25, no. 6, pp. 355-358, 1997.

[46] J. R. Verani, A. Seitz, R. H. Gilman et al., "Geographie variation in the sensitivity of recombinant antigen-based rapid tests for chronic Trypanosoma cruzi infection," American Journal of Tropical Medicine and Hygiene, vol. 80, no. 3, pp. 410-415, 2009.

[47] H. A. Lennox, D. A. Karcz, H. Tales, and M. El Masri, "Chagas disease: clinical overview and implications for nursing," Medsurg Nursing, vol. 16, no. 4, pp. 229-236, 2007.

[48] C. Bern, S. P. Montgomery, L. Katz, S. Caglioti, and S. L. Stramer, "Chagas disease and the US blood supply," Current Opinion in Infectious Diseases, vol. 21, no. 5, pp. 476-482, 2008.

[49] B. S. Schwartz, M. Paster, M. G. Ison, and P. V. ChinHong, "Organ donor screening practices for Trypanosoma cruzi infection among US organ procurement organizations," American Journal of Transplantation, vol. 11, no. 4, pp. 848851, 2011.

[50] G. A. Schmunis, "Trypanosoma cruzi, the etiologic agent of Chagas' disease: status in the blood supply in endemic and nonendemic countries," Transfusion, vol. 31, no. 6, pp. 547557, 1991.

[51] D. Dzib, V. P. Hernández, B. C. Ake, R. A. López, and V. M. Monteón, "Leukoreduction by centrifugation does not eliminate Trypanosoma cruzi from infected blood units," Vector-Borne and Zoonotic Diseases, vol. 9, no. 3, pp. 235-241, 2009.

[52] H. Moraes-Souza, J. O. Bordin, L. Bardossy, D. W. MacPherson, and M. A. Blajchman, "Prevention of transfusionassociated Chagas' disease: efficacy of white cell-reduction filters in removing Trypanosoma cruzi from infected blood," Transfusion, vol. 35, no. 9, pp. 723-726, 1995.

[53] D. A. Leiby, L. Nguyen, C. Procter, L. Townsend, and S. L. Stramer, "Impact of Trypanosoma cruzi phylogenetic lineage on transfusion transmission in the United States," Vox Sanguinis, vol. 99, supplement 1, p. 64, 2011.

[54] B. Custer, M. Agopova, H. Biswas, M. Busch, P. Tomasulo, and H. Kamel, "Do pre-donation questions identify donors at higher risk for Trypanosoma cruzi infection?” Transfusion, vol. 49, supplement 1, p. 17A, 2009.

[55] Centers for Disease Control and Prevention (CDC), "Chagas disease after organ transplantation-United States, 2001," Morbidity and Mortality Weekly Report, vol. 51, no. 10, pp. 210-212, 2002.

[56] P. Martin-Davila, J. Fortun, R. Lopez-Velez et al., "Transmission of tropical and geographically restricted infections during solid-organ transplantation," Clinical Microbiology Reviews, vol. 21, no. 1, pp. 60-96, 2008.

[57] M. J. Pinazo, B. Miranda, C. Rodríguez-Villar et al., "Recommendations for management of Chagas disease in organ and hematopoietic tissue transplantation programs in nonendemic areas," Transplantation Reviews, vol. 25, no. 3, pp. 91-101, 2011.

[58] Centers for Disease Control and Prevention (CDC), "Chagas disease after organ transplantation-Los Angeles, California, 
2006," Morbidity and Mortality Weekly Report, vol. 55, no. 29, pp. 798-800, 2006.

[59] L. A. Benvenuti, A. Roggério, N. V. Sambiase, A. Fiorelli, and M. D. L. Higuchi, "Polymerase chain reaction in endomyocardial biopsies for monitoring reactivation of Chagas' disease in heart transplantation: a case report and review of the literature," Cardiovascular Pathology, vol. 14, no. 5, pp. 265-268, 2005.

[60] R. B. Bestetti, P. M. Cury, T. A. D. Theodoropoulos, and D. Villafanha, "Trypanosoma cruzi myocardial infection reactivation presenting as complete atrioventricular block in a Chagas' heart transplant recipient," Cardiovascular Pathology, vol. 13, no. 6, pp. 323-326, 2004.

[61] R. W. Evans, "Ethnocentrism is an unacceptable rationale for health care policy: a critique of transplant tourism position statements," American Journal of Transplantation, vol. 8, no. 6, pp. 1089-1095, 2008.

[62] M. A. Shikanai-Yasuda and N. B. Carvalho, "Oral transmission of Chagas disease," Clinical Infectious Diseases, vol. 54, no. 6, pp. 845-852, 2012.

[63] M. A. Shikanai-Yasuda, C. B. Marcondes, L. A. Guedes et al., "Possible oral transmission of acute Chagas' disease in Brazil," Revista do Instituto de Medicina Tropical de Sao Paulo, vol. 33, no. 5, pp. 351-357, 1991.

[64] P. R. Benchimol Barbosa, "The oral transmission of Chagas' disease: an acute form of infection responsible for regional outbreaks," International Journal of Cardiology, vol. 112, no. 1, pp. 132-133, 2006.

[65] J. H. Maguire, R. Hoff, and A. C. Sleigh, "An outbreak of Chagas' disease in southwestern Bahia, Brazil," American Journal of Tropical Medicine and Hygiene, vol. 35, no. 5, pp. 931-936, 1986.

[66] A. A. Nóbrega, M. H. Garcia, E. Tatto et al., "Oral transmission of chagas disease by consumption of Açaí palm fruit, Brazil," Emerging Infectious Diseases, vol. 15, no. 4, pp. 653655, 2009.

[67] A. Y. Pinto, S. A. Valente, C. Valente Vda, A. G. Ferreira Junior, and J. R. Coura, "Acute phase of Chagas disease in the Brazilian Amazon region: study of 233 cases from Para, Amapa and Maranhao observed between 1988 and 2005," Revista da Sociedade Brasileira de Medicina Tropical, vol. 41, no. 6, pp. 602-614, 2008.

[68] L. M. Hernandez, A. N. Ramirez, Z. M. Cucunaba, and P. Zambrano, "Brote de Chagas agudo en Lebrija, Santander 2008," Revista Del Observatorio de Salud Publica de Santander, vol. 1, no. 28, 2009.

[69] B. A. De Noya, Z. Díaz-Bello, C. Colmenares et al., "Large urban outbreak of orally acquired acute chagas disease at a school in Caracas, Venezuela," Journal of Infectious Diseases, vol. 201, no. 9, pp. 1308-1315, 2010.

[70] H. Freilij and J. Altcheh, "Congenital Chagas' disease: diagnostic and clinical aspects," Clinical Infectious Diseases, vol. 21, no. 3, pp. 551-555, 1995.

[71] R. E. Gürtler, E. L. Segura, and J. E. Cohen, "Congenital transmission of Trypanosoma cruzi infection in Argentina," Emerging Infectious Diseases, vol. 9, no. 1, pp. 29-32, 2003.

[72] F. Torrico, C. Alonso-Vega, E. Suarez et al., "Maternal Trypanosoma cruzi infection, pregnancy outcome, morbidity, and mortality of congenitally infected and non-infected newborns in Bolivia," American Journal of Tropical Medicine and Hygiene, vol. 70, no. 2, pp. 201-209, 2004.

[73] S. Ciesielski, J. R. Seed, J. Estrada, and E. Wrenn, "The seroprevalence of cysticercosis, malaria, and Trypanosoma cruzi among North Carolina migrant farmworkers," Public Health Reports, vol. 108, no. 6, pp. 736-741, 1993.

[74] N. A. Salas, M. Cot, D. Schneider et al., "Risk factors and consequences of congenital Chagas disease in Yacuiba, south Bolivia," Tropical Medicine and International Health, vol. 12, no. 12, pp. 1498-1505, 2007.

[75] E. Hermann, C. Truyens, C. Alonso-Vega et al., "Congenital transmission of Trypanosoma cruzi is associated with maternal enhanced parasitemia and decreased production of interferon- $\gamma$ in response to parasite antigens," Journal of Infectious Diseases, vol. 189, no. 7, pp. 1274-1281, 2004.

[76] A. Moncayo, "Chagas disease: epidemiology and prospects for interruption of transmission in the Americas," World Health Statistics Quarterly, vol. 45, no. 2-3, pp. 276-279, 1992.

[77] E. A. De Castilho and G. R. Da Silva, "Maternal Chagas' infection and prematurity," Revista do Instituto de Medicina Tropical de Sao Paulo, vol. 18, no. 4, pp. 258-260, 1976.

[78] I. M. Hernandez-Matheson, R. F. Frankowski, and B. Held, "Foeto-maternal morbidity in the presence of antibodies to Trypanosoma cruzi," Transactions of the Royal Society of Tropical Medicine and Hygiene, vol. 77, no. 3, pp. 405-411, 1983.

[79] H. Schenone, C. M. Del Contreras, and J. M. Borgono, "Congenital Chagas' disease in Chile. Longitudinal study of reproductivity of chagasic and non-chagasic women and of some parasitological and clinical parameters from them and their corresponding children," Boletin Chileno de Parasitologia, vol. 40, no. 1-2, pp. 24-29, 1985.

[80] A. Pellegrino, "Migration from Latin America to Europe: trends and policy challenges," IOM Migration Research Series 16, International Organization for Migration, Geneva, Switzerland, 2004.

[81] M. C. Di Pentima, L. Y. Hwang, C. M. Skeeter, and M. S. Edwards, "Prevalence of antibody to Trypanosoma cruzi in pregnant hispanic women in Houston," Clinical Infectious Diseases, vol. 28, no. 6, pp. 1281-1285, 1999.

[82] Z. E. Yadon and G. A. Schmunis, "Congenital chagas disease: estimating the potential risk in the United States," American Journal of Tropical Medicine and Hygiene, vol. 81, no. 6, pp. 927-933, 2009.

[83] Y. Jackson, L. Gétaz, H. Wolff et al., "Prevalence, clinical staging and risk for blood-borne transmission of chagas disease among latin American migrants in Geneva, Switzerland," PLoS Neglected Tropical Diseases, vol. 4, no. 2, article e592, 2010.

[84] D. A. Leiby, M. C. H. Fucci, and R. J. Stumpf, “Trypanosoma cruzi in a low- to moderate-risk blood donor population: seroprevalence and possible congenital transmission," Transfusion, vol. 39, no. 3, pp. 310-315, 1999.

[85] P. Buekens, O. Almendares, Y. Carlier et al., "Mother-to-child transmission of chagas' disease in North America: why don't we do more?" Maternal and Child Health Journal, vol. 12, no. 3, pp. 283-286, 2008.

[86] A. L. S. Sgambatti De Andrade, F. Zicker, R. M. De Oliveira et al., "Randomised trial of efficacy of benznidazole in treatment of early Trypanosoma cruzi infection," The Lancet, vol. 348, no. 9039, pp. 1407-1413, 1996.

[87] S. S. Estani, E. L. Segura, A. M. Ruiz, E. Velazquez, B. M. Porcel, and C. Yampotis, "Efficacy of chemotherapy with benznidazole in children in the indeterminate phase of Chagas' disease," American Journal of Tropical Medicine and Hygiene, vol. 59, no. 4, pp. 526-529, 1998. 
[88] C. Bern, S. P. Montgomery, B. L. Herwaldt et al., "Evaluation and treatment of chagas disease in the United States: a systematic review," Journal of the American Medical Association, vol. 298, no. 18, pp. 2171-2181, 2007.

[89] H. S. Margolis, P. J. Coleman, R. E. Brown, E. E. Mast, S. H. Sheingold, and J. A. Arevalo, "Prevention of hepatitis B virus transmission by immunization: an economic analysis of current recommendations," Journal of the American Medical Association, vol. 274, no. 15, pp. 1201-1208, 1995.

[90] R. Storino and J. Milei, Enfermedad de Chagas, MosbyDoyma, Buenos Aires, Argentina, 1994.

[91] R. A. Guerri-Guttenberg, D. R. Grana, G. Ambrosio, and J. Milei, "Chagas cardiomyopathy: europe is not spared!," European Heart Journal, vol. 29, no. 21, pp. 2587-2591, 2008.

[92] L. V. Kirchhoff, J. R. Votava, D. E. Ochs, and D. R. Moser, "Comparison of PCR and microscopic methods for detecting Trypanosoma cruzi," Journal of Clinical Microbiology, vol. 34, no. 5, pp. 1171-1175, 1996.

[93] L. V. Kirchhoff, "Current concepts: American trypanosomiasis (Chagas' disease) - a tropical disease now in the United States," New England Journal of Medicine, vol. 329, no. 9, pp. 639-644, 1993.

[94] K. K. Stimpert and S. P. Montgomery, "Physician awareness of Chagas disease, USA," Emerging Infectious Diseases, vol. 16, no. 5, pp. 871-872, 2010.

[95] Medecins Sans Frontieres, Shortage of Benznidazole Leaves Thousands of Chagas Patients without Treatment, Medecins Sans Frontieres, 2011.

[96] M. Doty and B. Ives, "Quality of health care for Hispanic populations. Findings from the Commonwealth Fund 2001 health care quality survey," Tech. Rep. 526, New York, NY, USA, Commonwealth Fund, 2002.

[97] C. Di Girolamo, C. Bodini, and B. L. Marta, "Chagas disease at the crossroad of international migration and public health policies: why a national screening might not be enough," Euro Surveillance, vol. 16, no. 37, 2011.

[98] R. Briceno Leon and J. Mendez Galvan, "The social determinants of Chagas disease and the transformations of Latin America," Memórias do Instituto Oswaldo Cruz, vol. 102, supplement 1, pp. 109-112, 2007.

[99] R. Storino, "La cara oculta de la enfermeded de Chagas," Revista de la Federacion Argentina de Cardiologia, vol. 29, no. 31, 2000.

[100] C. Di Girolamo, B. L. Marta, A. Ciannameo et al., "Chagas disease in a non endemic country: a study in the district of Bologna (Italy). Multidisciplinary analysis of the disease in the Latin American migrant population," Annali di Igiene, vol. 22, no. 5, pp. 431-445, 2010.

[101] Platform for International Cooperation on Undocumented Migrants (PICUM), "Helath care in nowhereland: improving services for undocumented migrants in the EU," in Workpackage No. 6: The Voice of Undocumented Migrants. Undocumented Migrants' Health Needs and Strategies to Access Health Care in 17 EU countries, PICUM, Brussels, Belgium, June 2010.

[102] "Neglected parasitic infections in the United States," http:// www.cdc.gov/parasites/npi.html.

[103] "A new global effort to eliminate Chagas disease 2007," http://www.who.int/mediacentre/news/releases/2007/pr36/ en/index.html. 


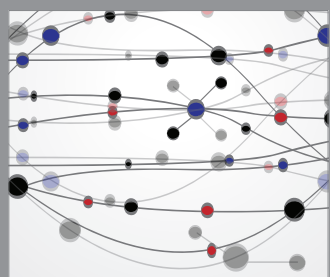

The Scientific World Journal
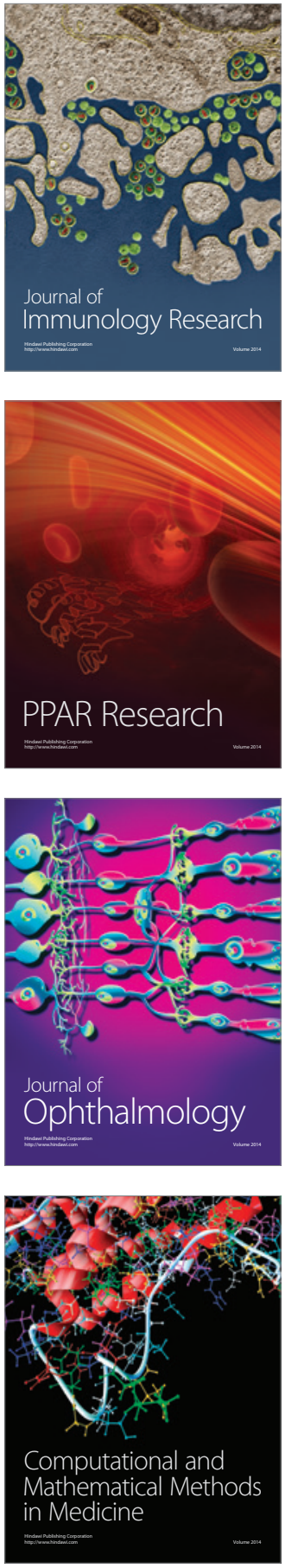

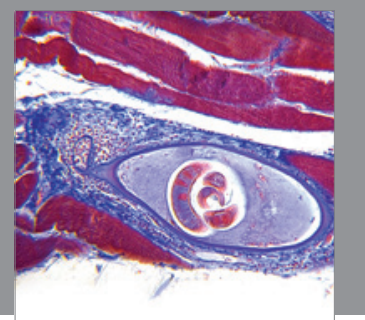

Gastroenterology

Research and Practice
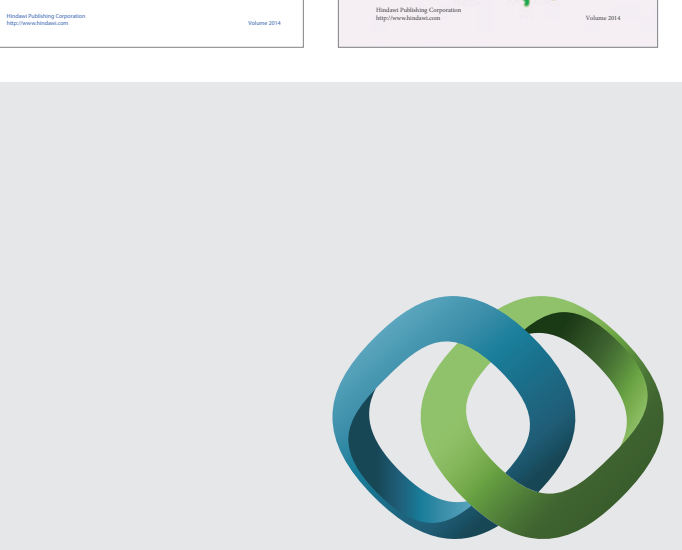

\section{Hindawi}

Submit your manuscripts at

http://www.hindawi.com
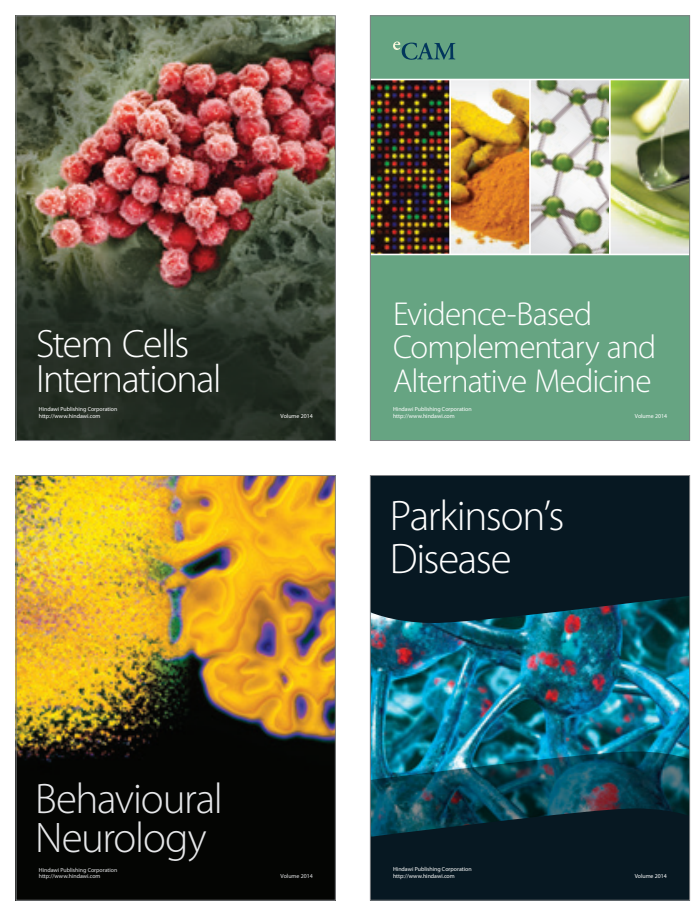

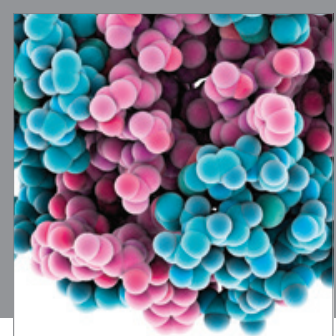

Journal of
Diabetes Research

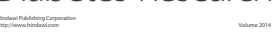

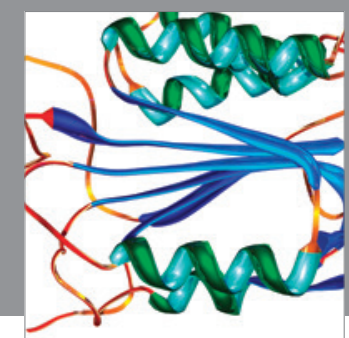

Disease Markers
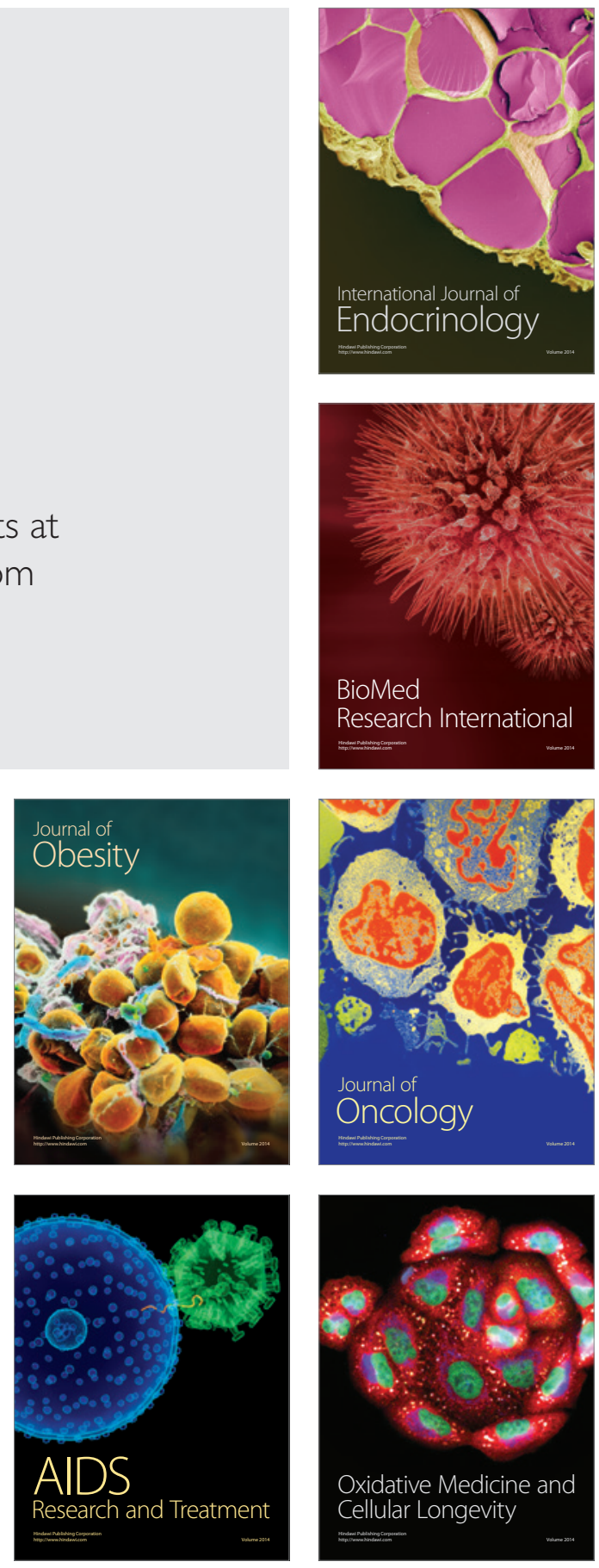